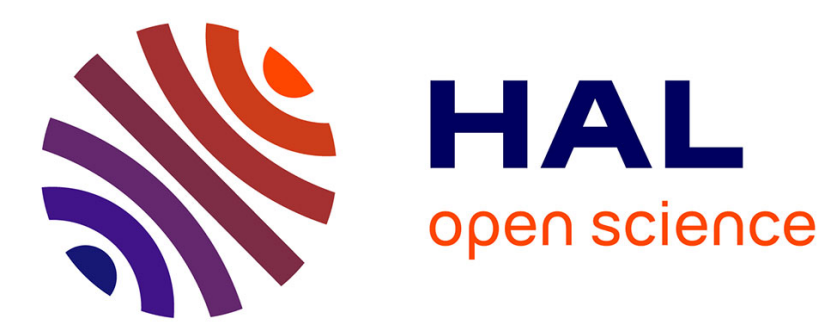

\title{
On intrinsic properties of fractal lattices and percolation clusters
}

\author{
J. Vannimenus
}

\section{To cite this version:}

J. Vannimenus. On intrinsic properties of fractal lattices and percolation clusters. Journal de Physique Lettres, 1984, 45 (22), pp.1071-1076. 10.1051/jphyslet:0198400450220107100 . jpa-00232451

\section{HAL Id: jpa-00232451 https://hal.science/jpa-00232451}

Submitted on 1 Jan 1984

HAL is a multi-disciplinary open access archive for the deposit and dissemination of scientific research documents, whether they are published or not. The documents may come from teaching and research institutions in France or abroad, or from public or private research centers.
L'archive ouverte pluridisciplinaire HAL, est destinée au dépôt et à la diffusion de documents scientifiques de niveau recherche, publiés ou non, émanant des établissements d'enseignement et de recherche français ou étrangers, des laboratoires publics ou privés. 
Classification

Physics Abstracts

$05.40-02.50-47.55 \mathrm{M}$

\title{
On intrinsic properties of fractal lattices and percolation clusters
}

\author{
J. Vannimenus \\ Groupe de Physique des Solides de l'Ecole Normale Supérieure, \\ 24, rue Lhomond, 75231 Paris Cedex 05, France
}

(Reçu le 20 août 1984, accepté le 21 septembre 1984)

\begin{abstract}
Résumé. - Certaines propriétés des réseaux fractals sont indépendantes du plongement Euclidien. Les implications de cette invariance sont discutées pour divers problèmes : conductivité d'amas de percolation, inégalités entre dimensions intrinsèques, coefficient de diffusion dans un milieu poreux en présence d'un écoulement.
\end{abstract}

\begin{abstract}
Certain properties of fractal lattices are independent of the Euclidean embedding. The implications of this invariance are discussed for various problems : the conductivity of percolation clusters, inequalities between intrinsic dimensions, and the diffusion coefficient in a flow through a porous medium.
\end{abstract}

\section{Introduction.}

The description of certain natural objects by fractals [1] is an idea which has recently met growing success in statistical physics. The study of physics on fractals sheds a new light on the notion of dimension : many properties, which for regular Euclidean lattices depend simply on the space dimension $d$, cannot be described by a single parameter on fractal lattices. In addition to the Hausdorff or fractal dimension $D$, which is defined through the mass contained within a given Euclidean distance, other independent numbers replace $d$ in various expressions. For instance, the spectral dimension [2] $\tilde{d}$ governs the density of states of low-energy excitations, diffusion and conduction properties, and renewal statistics. The spreading or connectivity dimension [3-6, 14] $\hat{d}$ describes the number of sites accessible in a given number of steps along the lattice, and controls the behaviour of localized impurity states [7].

It has been pointed out [8] that, among the properties of a fractal lattice, some are intrinsic, i.e. they do not depend on the way the lattice is embedded in Euclidean space. For instance, the spectral dimension of a chain is always $\tilde{d}=1$, whereas its fractal dimension may be $D=1$ (case of a straight line), $D=4 / 3$ (self-avoiding walk in two dimensions), $D=2$ (plane-filling Peano curve). The purpose of the present Letter is to emphasize that the notion of intrinsic property is a deep and useful one, which helps to formulate relations between various quantities and clarifies their content. It shows that some relations have a higher degree of generality than others, which in turn can only be accidental and due to some special feature of the particular embedding considered. This remark may be important for example when one tries to prove, or disprove, a proposed conjecture on critical exponents. 
The first part of this Letter is devoted to general considerations and a discussion of intrinsic properties of percolation clusters. The second part contains some specific applications to bounds and inequalities for intrinsic dimensions, and to diffusion of a dye molecule in a porous medium in the presence of a flow.

\section{Fractal invariance and intrinsic properties.}

Most fractal objects of physical interest possess a scaling invariance, meaning that their structure is self-similar under dilation (in practice, this will hold only in a wide range of scales between the lattice constant and a macroscopic length). Transformations of a scaling object which preserve its scaling invariance and its topological properties - such as the coiling of a linear chain into a Peano curve - may be called " fractal deformations". In general, they do not preserve the Hausdorff dimension $D$ of the original object, and one may obtain a whole set or " family " of objects of varying $D$ by applying various deformations. The physical properties which are invariant under such transformations are intrinsic and hold for the whole fractal family, whereas the other properties are extrinsic and belong to the particular realization considered.

A first important consequence is that a relation between intrinsic properties is valid for a fractal family, and it is sufficient to derive it for a particular member. Conversely, a relation between an intrinsic property and an extrinsic one must be a consequence of a specific feature of the family member for which it is derived. In many cases, the original derivation of a new relation involves extrinsic expressions, which may obscure the underlying invariance properties : it is therefore desirable to formulate the result in a way that explicitly displays fractal invariance when it exists.

We now recall some relevant definitions and general properties. The spectral and spreading dimensions $\tilde{d}$ and $\hat{d}$ may be respectively defined through the asymptotic behaviour of $S_{N}$, the average number of distinct visited sites in an $N$-step random walk on that fractal [2], and of $A_{N}$, the number of sites accessible within $N$ steps on that fractal from a given origin [3-5] :

$$
\begin{aligned}
& \frac{\ln S_{N}}{\ln N} \rightarrow \frac{\tilde{d}}{2} \quad(\text { if } \tilde{d} \leqslant 2) \\
& \frac{\ln A_{N}}{\ln N} \rightarrow \hat{d} .
\end{aligned}
$$

$$
N \rightarrow \infty
$$

These definitions make no reference to Euclidean distance between lattice sites, so $S_{N}$ and $A_{N}$ and the dimensions $\tilde{d}$ and $\hat{d}$ are independent of the embedding in space and are intrinsic properties. In some cases, like the Sierpinski gaskets, $\hat{d}$ is equal to the fractal $D$, but this is accidental : the Euclidean metrics happen to be equivalent to the natural metrics on the graph defined by these lattices [3]. A more detailed discussion of this point will be given later.

One of the most studied fractal objects is the incipient infinite cluster at the percolation threshold $p_{c}$, and the question arises naturally of knowing which of the related critical exponents are intrinsic. Near $p_{\mathrm{c}}$, the probability $P(p)$ that a site belongs to the infinite cluster behaves as $\left(p-p_{\mathrm{c}}\right)^{\beta}$. and the exponent $\beta$ is intrinsic since $P(p)$ is unaffected by a fractal deformation. The same result holds for the exponent $\gamma$ of the mean cluster mass, but not for the exponent $v$ of the connectivity length $\xi \sim\left(p-p_{c}\right)^{-v}$. Indeed, in a fractal transformation of the underlying lattice, the connectivity distance measured on a path along the cluster does not change, but the corresponding length measured in Euclidean space becomes

$$
\xi^{\prime}=\xi^{D / D^{\prime}},
$$

if the fractal dimension of the cluster is changed from $D$ to $D^{\prime}$. The exponent $v$ is modified according to

$$
v^{\prime}=v \frac{D}{D^{\prime}},
$$


so the combination $D v$ is invariant, as originally pointed out in reference [8]. This result may be also obtained directly by considering the typical mass $M^{*}$ (i.e. number of sites) of a large cluster, just below $p_{\mathrm{c}}$ :

$$
M^{*} \sim\left(p-p_{\mathrm{c}}\right)^{-D v} .
$$

More generally, properties involving a mass or a time, but no Euclidean distance, are intrinsic.

It is instructive in this respect to consider the conductivity $\Sigma \sim\left(p-p_{c}\right)^{t}$. As discussed by several authors [2], the time $\tau$ needed for a random walker to diffuse on the typical length, above $p_{\mathrm{c}}$, is given by

$$
\tau^{-1} \sim\left(p-p_{\mathrm{c}}\right)^{2++t-\beta} .
$$

This time is not affected by a fractal deformation, so its exponent is intrinsic : this shows that the combination $(t+2 \mathrm{v})$ is intrinsic, though $t$ itself is not. Using exact relations between $t$ and other exponents [2], one can write a relation where both sides are explicitly intrinsic :

$$
t+2 v=\beta+2 D v / \tilde{d}
$$

It is interesting to observe that this combination of exponents varies little with $d: t+2 v=4$ for $d \geqslant 6,3.7 \pm 0.1$ for $d=3[9]$ and $3.96 \pm 0.01$ for $d=2[10]$.

The conductance $g$ of a fractal cluster is usually expressed as a function of its linear size $L$ :

$$
g \sim L^{\beta_{\mathbf{L}}},
$$

with $\beta_{\mathrm{L}}=D(\tilde{d}-2) / \tilde{d}$. This can be written in intrinsic form, in terms of the mass $M$ of the cluster :

$$
g \sim M^{-\phi}, \quad \phi=(2-\tilde{d}) / \tilde{d} .
$$

The much discussed Alexander-Orbach conjecture [2], $\tilde{d}=4 / 3$ for percolation, corresponds to $g \sim M^{-1 / 2}$ for all $d \geqslant 2$.

\section{Bounds on intrinsic dimensions.}

The various dimensions discussed above are not simply related to one another a priori, but there exist some general inequalities between them. Let us consider the shortest path through an isotropic fractal lattice. If this path has $2 L_{\min }$ steps, the number $A_{L_{\min }}$ of accessible sites in $L_{\min }$ steps will be a finite fraction of the total number of sites $M$, unless most of the mass is contained in very long filaments dangling off the backbone of the lattice (i.e. its doubly connected component). Excluding this case, one has for a fractal of Euclidean linear size $2 L[11,12]$ :

$$
A_{L_{\min }} \sim M \Rightarrow L_{\min }^{\hat{d}} \sim L^{D} .
$$

The shortest path $L_{\min }$ has a power law dependence on $L[13,14]$, which may be written :

$$
L_{\min } \sim L^{\delta},
$$

so the exponent $\delta$ is given by

$$
\delta=D / \hat{d}
$$

which is well verified numerically for the infinite percolation cluster $[11,12]$ (e.g. for $d=2$ : $\left.D \simeq 1.896, \hat{d}=1.65-1.7, \delta=\gamma_{\min } / v \simeq 1.12 \pm 0.02[14]\right)$.

Now, the Euclidean distance between two lattice sites is never larger than the shortest path along the lattice, whatever the fractal deformation $\mathcal{F}$ considered, so :

$$
\hat{d} \leqslant \operatorname{Min}_{\{\mathfrak{F}\}} D \text {. }
$$


On the other hand, the spectral dimension obeys the inequality [2] $\tilde{d} \leqslant D$. If there exists a deformation $\mathscr{F}_{0}$ such that $\hat{d}=D\left(\mathcal{F}_{0}\right)$, this inequality holds for $D\left(\mathscr{F}_{0}\right)$ and one can conclude that

$$
\tilde{d} \leqslant \hat{d} \leqslant D,
$$

for all members of the fractal family. Relation (13) has been first proposed by Rammal et al. [3]. who conjectured that it holds for any fractal. Objects for which $\hat{d}=D$, such as the Sierpinski gaskets, appear in the light of the above discussion as the natural realizations of their family, in the sense of maximum geometrical simplicity.

Another inequality may be obtained by generalizing a relation derived by Witten and Kantor [15] for branched objects without loops. By considering the conductance between the centre of such a branched fractal and a large surrounding sphere, these authors have shown that the exponent $\delta$ of the shortest path is then given by :

$$
\delta=D\left(\frac{2}{\tilde{d}}-1\right) .
$$

(In other words, $\delta=-\beta_{\mathrm{L}}$, see Eq. (7)). This gives, for branched fractals, using equation (11),

$$
\hat{d}=\tilde{d} /(2-\tilde{d}) \text {. }
$$

In the case of a general isotropic fractal, let us choose an origin A on the backbone and draw a large sphere of origin $\mathrm{A}$ and radius $L$. For every point $\mathrm{P}$ of the sphere connected to $\mathrm{A}$, keep the shortest path on the fractal between $A$ and $P$ (choose one of them if there are several such paths) : the ensemble $\mathrm{C}$ of shortest paths is a subset of the backbone, and is branched in the sense of Witten and Kantor, so relation (14) holds for C. Since the conductance $g_{\mathrm{C}}$ of C is necessarily smaller than the conductance $g$ of the full fractal (or its backbone), one deduces from (7) for the conductance $g(L)$

$$
D\left(\frac{2}{\tilde{d}}-1\right) \leqslant D_{\mathrm{C}}\left(\frac{2}{\tilde{d}_{\mathrm{C}}}-1\right) .
$$

The right-hand side of this equation is just $\delta_{\mathrm{C}}$, according to equation (14), which is by construction equal to $\delta$ for the full fractal. Using $\delta=D / \hat{d}$ and dividing both sides by $D$, one finally obtains :

$$
\hat{d}(2 / \tilde{d}-1) \leqslant 1,
$$

which can be combined with (13) to write

$$
\hat{d} \geqslant \tilde{d} \geqslant 2 \frac{\hat{d}}{1+\hat{d}} .
$$

For the infinite percolation cluster in two dimensions, the best available numerical results [3, 5] give $\hat{d}=1.65-1.7, \tilde{d}=1.32 \pm 0.01$, and $2 \hat{d} /(1+\hat{d})=1.25 \pm 0.01$, whereas for the backbone $\hat{d}_{\mathrm{B}}=1.44 \pm 0.03[10], \tilde{d}_{\mathrm{B}}=1.25 \pm 0.01[16]$ and $2 \hat{d}_{\mathrm{B}} /\left(1+\hat{d}_{\mathrm{B}}\right)=1.19 \pm 0.02$. For $d=3$, $\hat{d}=1.83 \pm 0.02[5,11]$ and $2 \hat{d} /(1+\hat{d})=1.29 \pm 0.01$ is very close to $\tilde{d} \simeq 4 / 3$. The new lower bound in (17) is seen to be rather strong in all these cases.

\section{Diffusion in a flow through a porous medium.}

The properties of isotropic random walks on percolation clusters and fractal objects have been intensively studied, and anomalous isotropic diffusion on fractals is now well understood. By contrast, the problem of biased diffusion in a random medium remains of current interest [17, 18], in connection with the diffusion of dye molecules or contaminants through a porous medium, in the presence of a steady flow $[19,20]$. 
A detailed description of flow in a porous medium is quite intricate, and here only the regime just above an injection threshold will be considered. In this regime, the injected non-wetting fluid partially fills the porous matrix, and the network of filled pores may be reasonably described as an infinite percolation cluster above $p_{\mathrm{c}}$ [19]. Diffusion properties then depend on the relative importance of convection along the backbone and molecular diffusion in the dead ends. By analysing these two contributions for a small pressure difference $\left(p-p_{c}\right)$ above the injection threshold, de Gennes [19] has argued that the longitudinal diffusion coefficient $D_{\|}$is given by :

$$
D_{\|} \sim \xi^{2} \frac{\bar{U}^{2}}{D_{\mathrm{a}}} \sim\left(p-p_{\mathrm{c}}\right)^{\omega},
$$

with

$$
\omega=t-\beta-2 v .
$$

In expression (18), $D_{\|}$corresponds to the diffusion of the dye along the average fluid flow, $\bar{U}$ is the average velocity of a fluid particle, measured in the flow direction, $\xi$ is the correlation length for percolation and $D_{\mathrm{a}}$ is the diffusion coefficient in the absence of flow.

To study the intrinsic aspects of diffusion, it is useful to note that a diffusion coefficient has the dimension of (length) $)^{2} /\left(\right.$ time), so the combination $D_{\|} / \xi^{2}$ is the inverse of a time. Following the discussion given above for the conductivity, this quantity is expected to be intrinsic, and its exponent $(\omega+2 v)$ should be given by an intrinsic expression. This is not the case for equation (19), since $t$ is not intrinsic as discussed earlier.

The crucial point in the derivation of equation (18) is that the velocity involved is the average velocity $\bar{U}$ over all possible positions of the dye particle, including dead ends, and not the average velocity $\bar{U}_{\mathrm{B}}$ for particles on the backbone, as one might naively expect. However, the local velocity $\mathbf{U}_{\mathbf{B}}$ of the dye appears at an intermediate stage in the calculation : the magnitude of this local velocity can be defined with respect to the local pore structure, so it does not depend on the embedding in Euclidean space and is an intrinsic quantity. In practice, this means that the shortest path length through the solid should be used in the calculation of average velocities, instead of the physical length of the system.

This remark leads to replace $\bar{U}$ by $\bar{U}^{\prime}=\bar{U}\left(L_{\min } / L\right)$ in equation (18). Noting that

$$
L_{\min } \sim L\left(p-p_{\mathrm{c}}\right)^{-\lambda},
$$

with $\lambda=v(\delta-1)$ for consistency with equation (10), one obtains

$$
D_{\|} / \xi^{2} \sim(\Delta p)^{\psi},
$$

where

$$
\psi=t-\beta+2 v-2 v D / \hat{d}
$$

is now an intrinsic exponent, in agreement with the general discussion. Finally, the critical exponent of the diffusion coefficient is given in the present theory by

$$
\omega=t-\beta-2 v D / \hat{d} \text {. }
$$

Using recent numerical values for $t / v[19]$ and $\hat{d}[5,11]$ in three dimensions, expression (12) gives $\omega / v \simeq-0.3$, whereas the new result (20) predicts $\omega / v \simeq-1$, hence a much stronger divergence. Numerical evidence for a rather strong divergence of $D_{\|}$has been recently found by Sahimi et al. [21], for two-phase flow near the percolation threshold. This is not entirely conclusive, because their model is somewhat different from the present one. Also, the region where the asymptotic form (21) is valid may be narrow and difficult to study on samples of limited size. The same caveat holds for the application to physical porous systems, and the tortuosity effect described by equation (23) might be difficult to observe in practice. 


\section{Conclusion.}

The common theme in the questions considered here is the general idea that intrinsic properties of fractal objects should be given special attention. Our presentation has been sketchy and illustrative, but the notion of invariance under scaling transformations, which has been hinted to, is probably deep.

\section{Acknowledgments.}

I thank E. Guyon for pointing out reference [20] to my attention, and G. Toulouse for fruitful discussions on intrinsic properties and a critical reading of the manuscript.

\section{References}

[1] Mandelbrot, B. B., The fractal geometry of Nature (Freeman, San Francisco) 1982.

[2] Alexander, S., Orbach, R., J. Physique Lett. 43 (1982) L-625.

Rammal, R., Toulouse, G., J. Physique Lett. 44 (1983) L-13.

Gefen, Y., Aharony, A., Alexander, S., Phys. Rev. Lett. 50 (1983) 77.

[3] Rammal, R., Angles d'Auriac, J. C., Benoit, A., J. Phys. A 17 (1984) L491.

[4] Vannimenus, J., Nadal, J. P., Martin, H., J. Phys. A 17 (1984) L351.

[5] Havlin, S., Nossal, R., J. Phys. A 17 (1984) L427.

[6] Grassberger, P., Math. Biosci. 62 (1983) 157.

[7] LeVY, Y., Souillard, B., private communication.

[8] Rammal, R., Toulouse, G., Vannimenus, J., J. Physique 45 (1984) 389.

[9] Derrida, B., Stauffer, D., Herrmann, H. J., Vannimenus, J., J. Physique Lett. 44 (1983) L701.

[10] Hong, D. C., Havlin, S., Herrmann, H. J., Stanley, H. E., Phys. Rev. B 30 (1984) 4083.

[11] Angles d'Auriac, J. C., Benoit, A., Harris, A. B., Rammal, R., preprint.

[12] Stanley, H. E., J. Stat. Phys. 35 (1984) to appear.

[13] Middlemis, K. M., Whittington, S. G., Gaunt, D. S., J. Phys. A 13 (1980) 1835.

[14] Pike, R., Stanley, H. E., J. Phys. A 14 (1981) Ll69.

Hong, D. C., Stanley, H. E., J. Phys. A 16 (1983) L475, L525.

[15] Witten, T. A., Kantor, Y., Phys. Rev. B 30 (1984) 4093.

[16] Puech, L., Rammal, R., J. Phys. C 16 (1983) L1197.

[17] Derrida, B., LuCK, J. M., Phys. Rev. B 28 (1983) 7183.

[18] Aronovitz, J. A., Nelson, D. R., Phys. Rev. A 30 (1984) 1948.

[19] De Gennes, P. G., J. Fluid Mech. 136 (1983) 189.

[20] The discussion does not apply to the diffusion of charged particles in an electric field, which is a tricky problem. See, e.g., PANDey, R. B., Phys. Rev. B 30 (1984) 489.

[21] Sahimi, M., Davis, H. T., SCriven, L. E., Chem. Eng. Commun. 23 (1983) 329. 